\title{
Procesos de control ejecutivo en la enfermedad de Alzheimer
}

\author{
$\mathrm{M}^{\mathrm{a}}$ Rosario García-Viedma ${ }^{1 *}$, Sara Fernández-Guinea ${ }^{2}$ y Rafael Martos-Montes ${ }^{1}$ \\ ${ }^{1}$ Departamento de Psicología. Universidad de Jaén (España) \\ ${ }^{2}$ Departamento de Psicología Básica (Procesos Cognitivos II). Facultad de Psicología. Universidad Complutense de Madrid (España)
}

\begin{abstract}
Resumen: El control ejecutivo (CE) es un mecanismo complejo compuesto por distintos subprocesos y vinculado al funcionamiento del lóbulo frontal. En los últimos años se ha generado una gran cantidad de investigación sobre los déficit de control ejecutivo (CE) en pacientes con enfermedad de Alzheimer (EA). Este análisis resulta de gran interés porque, por una parte, estudios recientes han observado precisamente alteraciones en regiones frontales en estos pacientes, y por otra parte, porque sus manifestaciones clínicas y conductuales en el inicio de la enfermedad están relacionadas con la afectación del CE. En este artículo se revisa detalladamente la evidencia existente sobre la afectación de los subprocesos del CE al inicio de la EA, determinando si habría un deterioro generalizado o disociaciones entre los componentes, así como sus posibles implicaciones básicas y aplicadas.
\end{abstract}

Palabras clave: Alzheimer; control ejecutivo; neuropsicología; red ejecuti-

Title: Executive control processes in Alzheimer's disease.

Abstract: It has been recently proposed that executive control (EC) is composed by different subprocesses. In recent times, EC deficits in Alzheimer's disease patients (AD) have been widely investigated. This analysis is very interesting because, on the one hand, recent studies have observed frontal lobe alterations on these demented patients, and on the other hand, Alzheimer's disease clinical and behavioural manifestations could be explained by the involvement of EC. This paper discusses in detail the possible involvement of $\mathrm{EC}$ subprocesses at the beginning of $\mathrm{AD}$, determining if there would be a widespread decline or dissociations between components and their basic and clinical implications.

Keywords: Alzheimer; executive control; neuropsychology; executive network. va.

\section{Introducción}

El control ejecutivo (CE) es un mecanismo complejo que implica varias operaciones relacionadas con el control y la regulación de la conducta. Este mecanismo depende de un sistema neuronal distribuido en el que destaca el papel del córtex prefrontal (CPF), el cual tiene importantes conexiones córtico-corticales (con córtex asociativo y paralímbico) y córtico-subcorticales (principalmente con los ganglios basales, el tálamo y el hipocampo) (Cummings, 1993; Faglioni, 1999; Fuster, 1997; Karatekin, Lazareff y Asarnow, 2000; Quintana y Fuster, 1999). De esta forma, el correcto funcionamiento del CE depende de regiones corticales, tanto anteriores como posteriores, y subcorticales.

$\mathrm{El} \mathrm{CE}$ ha sido explorado extensamente en diversas patologías, como por ejemplo en tumores cerebrales, traumatismos craneoencefálicos, enfermedades cerebrovasculares, la enfermedad de Parkinson, la esclerosis múltiple, etc. (Fontaine, Azouvi, Remy, Bussel, y Samson, 1999; Brown y Marsden, 1998; Foong, Rozewicz, Quaghebeur, Davie y Kartsounis, 1997; Leskela, Heitanen, Kalska, Ylikoski y Pdijasvaara, 1999). Del mismo modo, el análisis del funcionamiento ejecutivo en la enfermedad de Alzheimer (EA) ha sido objeto de un gran interés. Diversos estudios muestran que estos sujetos tienen dificultades a la hora de llevar a cabo actividades de la vida diaria (AVD) que demandan al CE, y muestran alteraciones neuropsicológicas relacionadas con el mismo desde estadios relativamente tempranos de la enfermedad (Alberoni, Baddeley, Della Sala, Logie y Spinnler, 1992; Amieva, Phillip, Della Sala y Henry, 2004; Belleville, Rouleau y Van der Linden, 2003; Collette y Van der Linden, 2002; Duchek, Hunt, Ball, Buckles y Morris, 1998; Martini,

* Dirección para correspondencia [Correspondence address]: $\mathrm{M}^{\mathrm{a}}$ Rosario García-Viedma. Departamento de Psicología. Universidad de Jaén. 23071 Jaén (España). E-mail: mrgarcia@ujaen.es
Benke, Domahs y Delazer, 2003; Perry y Hodges, 1999). Además, recientes investigaciones neuropatológicas, electrofisiológicas y de neuroimagen han mostrado que, además de otras áreas más posteriores y subcorticales, se encuentran afectadas áreas anteriores como el córtex prefrontal, que están implicadas en las manifestaciones clínicas de la EA desde etapas muy iniciales (Azari et al., 1992; Berendse, Verbundt, Scheltens, van Dijk y Jonkman, 2000; Delbeuck, Collette y Van der Linden, 2007; Horwitz, Grady, Sclageter, Duara y Rapoport, 1987; Grady, Furey, Pietrini, Horwitz y Rapoport, 2001; Wang et al., 2007).

Puesto que la evidencia pone de manifiesto que los déficit de CE en los inicios de la EA tiene importantes repercusiones de cara a la comprensión de los cambios cognitivos y conductuales que tienen lugar en la enfermedad, resulta interesante revisar la literatura existente sobre la afectación de los subprocesos del CE al inicio de la EA, con el objetivo de poder determinar si se da un deterioro generalizado o si es posible hablar de disociaciones entre los distintos procesos de CE.

\section{El control ejecutivo}

El CE es un constructo cognitivo complejo que se caracteriza por una gran diversidad en cuanto a las descripciones que se han hecho de él. Así, se han propuesto distintos modelos que tienen en común la idea de que este mecanismo permite controlar y regular la conducta a través de diversos procesos cognitivos, siendo necesario establecer unidades manejables (Tirapu-Ustárroz, García-Molina, Luna-Lario, Roig-Rovira, 2008a,b; Tirapu-Ustárroz, Muñoz-Céspedes y PelegrínValero, 2002).

Entre las aproximaciones teóricas propuestas, el modelo del ejecutivo central (EC) de Baddeley es uno de los modelos asociados al estudio del CE de mayor influencia (Badde- 
ley, 1996). El EC se presenta como un controlador atencional que lleva a cabo cuatro funciones: 1) coordinar la realización simultánea de más de una tarea, b) cambiar las estrategias de recuperación de la información, c) seleccionar la información relevante y eliminar la irrelevante y d) actualizar la información de la MCP. De forma similar, Miyake et al. (2000) llevan a cabo un análisis factorial y describen tres procesos que definen como: a) control inhibitorio o resistencia a la interferencia, b) capacidad para cambiar entre tareas o configuraciones mentales, y c) actualización de la memoria de trabajo.

En la Tabla 1 se presentan las propuestas de Baddeley (1996) y de Miyake et al. (2000) referentes a los componentes de control, y sobre las cuales puede establecerse un paralelismo. De esta forma, se puede hablar de cuatro operaciones principales de control: a) control inhibitorio, b) coordinación de diferentes tareas o canales de procesamiento, c) cambio de configuración mental, y d) actualización de la memoria de trabajo.

Tabla 1. Operaciones de control ejecutivo establecidas por Baddeley (1996) y por Miyake et al. (2000).

Baddeley (1996)
mación irrelevante
Coordinación de la reali-
$\begin{aligned} & \text { zación simultánea de } \\ & \text { más una tarea } \\ & \text { Cambio de estrategias de }(2000) \\ & \text { recuperación o planes de } \\ & \text { acción } \\ & \text { Activación de informa- } \\ & \text { ción de la memoria a } \\ & \text { largo plazo }\end{aligned}$

El control inhibitorio hace referencia a una inhibición deliberada, por tanto no automática, de información irrelevante para nuestros objetivos o de respuestas prepotentes. En la literatura cognitiva y neuropsicológica se han utilizado diversas tareas para valorar el control inhibitorio, como son: el paradigma de priming negativo (Neill, Valdes y Terry, 1995; Tipper, 1985), el test stroop (Stroop, 1935), la tarea Hayling (Burgess y Shallice, 1996), la tarea Simon (Simon, 1969), los tests go-no go y stop signal, y las tareas antisacádicas.

La capacidad para coordinar la realización simultánea de distintas tareas implicaría la habilidad de conjugar la información procedente de las distintas tareas o canales de procesamiento. Esta capacidad puede ser valorada mediante tareas que requieren el procesamiento de múltiples características o rasgos simultáneamente, como las tareas duales, que son el paradigma más utilizado para su investigación.

El cambio de configuración mental puede entenderse como la capacidad para cambiar "las estrategias de recuperación o planes de acción" (Baddeley, 1996; Baddeley, Baddeley, Bucks y Wilcox, 2001) o como la "habilidad para cambiar entre múltiples tareas, operaciones o configuraciones mentales" (Miyake et al., 2000). El cambio de una tarea a otra re- quiere del CE porque el sistema cognitivo debe ser "reconfigurado", es decir, el procedimiento o plan requerido para realizar la primera tarea debe abandonarse y activar el necesario para la tarea nueva. En cualquier caso, se enfatiza la capacidad de cambio de configuraciones mentales, ya que éstas variarán en función de la tarea que haya que realizar en cada momento. Esta capacidad es fundamental para poder reaccionar de forma eficaz a la estimulación ambiental, que cambia continuamente. Esta función se ha estudiado clásicamente con el paradigma de cambio de tarea (Allport, Styles y Hsieh, 1994; Kiesel et al., 2010; Logan, 2003; Monsell; 2003; Monsell y Mizon, 2006; Pashler, 2000; Rogers y Monsell, 1995).

Finalmente, la actualización de la memoria de trabajo incluye la "capacidad para activar información de la memoria a largo plazo" de Baddeley (1996) y la "actualización de la memoria de trabajo" de Miyake et al. (2000). En las dos propuestas el aspecto común es el mantenimiento y la manipulación activa de la información en la memoria de trabajo. Por tanto, esta capacidad implicaría algo más que el simple mantenimiento de la información, involucrando una manipulación dinámica de los contenidos de la memoria de trabajo, de tal forma que se modifica continuamente la información de acuerdo a la nueva información que llega. Esta función interviene en un gran número de AVD, como el aprendizaje y organización de información adquirida recientemente. Clásicamente, ha sido explorada con el paradigma "running memory", aunque también puede valorarse con otras tareas cognitivas, como el paradigma " $n$-back" y las tareas de amplitud de memoria de trabajo (Daneman y Carpenter, 1980).

Desde un punto de vista neurofisiológico, se ha intentado determinar las relaciones entre las funciones del CE y las estructuras y redes neuronales. Una de las principales propuestas las ha vinculado con la con la red ejecutiva del modelo de Posner y Petersen (1990), entre otros modelos (p.e. Damasio, 1998; Fuster, 2002; Koechlin, Ody y Kouneiher, 2003; Miller y Cohen, 2001; Shimamura, 2002). Esta red está formada anatómicamente por áreas de la corteza prefrontal medial, incluyendo la parte anterior del giro cingular, el área motora suplementaria superior y los ganglios basales. No obstante, esta red mantiene importantes interconexiones con otras dos redes, la red de alerta y la red de orientación, que se encuentran asentadas en regiones posteriores y subcorticales. La red de orientación está formada por el córtex parietal posterior, los núcleos pulvinar y reticular del tálamo y los colículos superiores; y la red de alerta está constituida por las proyecciones norepinefrinérgicas procedentes del locus coreuleus a la corteza cerebral frontal y parietal, y se encuentra lateralizada en el hemisferio derecho (Estévez-González, García-Sánchez y Junqué, 1997; Goldman-Rakic, 1988). Además, los estudios de neuroimagen que han intentado determinar las regiones cerebrales implicadas en las operaciones de CE han informado que participan tanto regiones anteriores (p.e. D’Esposito et al., 1995; Konishi et al., 1998; Leung, Skudlarski, Gatenby, Peterson y Gore, 2000; Van der Linden et. al., 1999) como posteriores y subcorticales (p. e. 
Bunge, Klinberg, Jacobse y Gabrieli, 2000; Jonides et al., 1997; Taylor, Kornblum, Lauber, Minoshima y Koeppe, 1997; Wilkinson, Halligan, Marshall, Büchel y Dolam, 2001). De esta forma, se ha señalado que las funciones del CE deben comprenderse en términos de interrelaciones entre redes de áreas cerebrales (Botvinick, Braver, Carter, Barch, y Cohen, 2001; Yeung, Cohen y Botvinic, 2004; Collette y Van der Linden, 2002).

\section{El control ejecutivo en la EA}

Como hemos comentado previamente, el correcto funcionamiento del EC requiere de la participación de regiones corticales anteriores y posteriores, y subcorticales, destacando el papel del córtex prefrontal. Asimismo, se ha apuntado el papel de regiones frontales en las manifestaciones clínicas de la EA desde etapas tempranas. De esta forma, los estudios neuropatológicos, electrofisiológicos y de neuroimagen en la EA han proporcionado evidencia a favor de la alteración de las interacciones entre diferentes áreas cerebrales, entre las que se encuentran regiones frontales.

Por ejemplo, diversos estudios neuropatológicos han puesto de manifiesto que las áreas de asociación son particularmente vulnerables a la presencia de ovillos neurofibrilares, que predominan en las áreas de asociación de los lóbulos temporal, parietal y frontal (Delbeuck et al., 2007). Con la técnica de magnetoencefalografía se ha observado una disminución de los valores de coherencia electroencefalográfica de todas las frecuencias analizadas, siendo mayor en las medidas de coherencia fronto-parietal (Berendse et al., 2000). Otra técnica utilizada es la tomografía por emisión de positrones. Horwitz et al. (1987) calcularon los coeficientes de correlación entre los valores de consumo de glucosa para estimar la asociación funcional entre áreas cerebrales, y observaron un número de correlaciones fronto-parietales reducido en los pacientes con EA con respecto al grupo control en estado de reposo. Azari et al. (1992) también mostraron interacciones neocorticales más débiles, especialmente entre áreas paralímbicas y áreas de asociación parietal y frontal. Grady et al. (2001) utilizan como medida $\mathrm{H}_{2} \mathrm{O}^{15}$ y encuentran una interacción funcional disminuida del córtex prefrontal derecho con el hipocampo derecho y el córtex visual, lo que apunta hacia una desconexión antero-posterior. Por último, utilizando la resonancia magnética funcional, Wang et al. (2007) encuentran alteraciones en las conexiones funcionales entre el hipocampo y otras áreas cerebrales como el córtex prefrontal medial, el córtex cingular anterior ventral y el posterior.

Además de estos datos, también se ha observado que los pacientes presentan manifestaciones clínicas que se han vinculado a problemas relacionados con el CE desde los primeros momentos de la EA, como son las dificultades para realizar AVD, para conducir o alteraciones en la comprensión del lenguaje (Duchek et al., 1998; Perry y Hodges, 1999; Faust, Balota, Duchek, Gernsbacher y Smith, 1997). A continuación analizaremos detalladamente la evidencia neurop- sicológica con respecto a las operaciones de CE en los inicios de la EA.

\section{Control inhibitorio}

Como se ha señalado previamente, diversos paradigmas se han utilizado para valorar esta operación de control en la EA. El paradigma de priming negativo mide la eficacia con la que un individuo inhibe información distractora para centrar la atención en la relevante. En este paradigma se presentan como targets, estímulos distractores que recientemente han tenido que ser ignorados. En un experimento típico los estímulos son dos dibujos (o palabras o letras) superpuestos. Uno es de color rojo, que es el que hay que nombrar; y otro de color verde que hay que ignorar. Para valorar el priming negativo, se muestra a los participantes un ensayo prime con un dibujo target pintado en rojo, junto con un dibujo distractor pintado en verde. A continuación tiene lugar un ensayo de prueba en el que el dibujo target rojo que tiene que ser nombrado es ahora el dibujo distractor del ensayo prime. Normalmente se observa un incremento en la latencia de respuesta en este tipo de ensayo comparado con los ensayos prueba, y se piensa que refleja la inhibición de representaciones internas de la información distractora en el ensayo prime (Houghton y Tipper, 1994). Se asume que la selección del target ocurre en parte por medio de la inhibición de representaciones internas de los distractores que compiten por el control de la acción. Si las representaciones de los distractores son inhibidas, entonces el procesamiento de un estímulo cuya representación ha sido inhibida recientemente será deficiente. Son pocos los estudios que han explorado los procesos inhibitorios utilizando este paradigma, y los resultados no son consistentes. Sullivan, Faust y Balota (1995) utilizan como estímulos dibujos y palabras y comparan el efecto de priming negativo en personas mayores sanas y en pacientes con EA. Los pacientes no muestran evidencia de priming negativo. Igualmente, Amieva et al. (2002) tampoco encuentran evidencia de este efecto en el grupo de pacientes con EA utilizando como estímulos dibujos. Por el contrario, Langley, Overmier, Knopman y Prod'homme (1998) encontraron una ejecución similar en jóvenes, mayores y pacientes con EA utilizando como estímulos letras. Se ha propuesto que las diferencias metodológicas podrían explicar las discrepancias en los resultados. Mientras Sullivan et al. (1995) y Amieva et al. (2002) utilizan estímulos más complejos (palabras y dibujos vs. letras) que se presentan de forma informatizada durante intervalos de tiempo cortos, Langley et al. (1998) muestran en tarjetas listas de letras hasta que el participante da sus respuestas. Posteriormente se han desarrollado tareas de priming negativo espacial, en la que los participantes deben identificar la localización espacial del target. En la tarea de localización espacial "X-O" (Tipper, Brehaut y Driver, 1990), los participantes presionan una tecla para indicar la localización espacial del target "O", mientras ignoran el distractor " $\mathrm{X}$ ". En esta tarea los tiempos de respuesta incrementan cuando el target "O" se presenta en la localiza- 
ción del distractor " $\mathrm{X}$ " en el ensayo precedente. Simone y Baylis (1997) muestran que los pacientes con EA presentan menos priming negativo que los adultos sanos, quienes producen niveles similares a los adultos jóvenes [véase Verhaeghen y De Meersman (1998) para un meta-análisis]. Utilizando una tarea de priming espacial, Vaughan, Hughes, Jones, Woods y Tipper (2006) también han encontrado que los pacientes con EA no muestran el efecto de priming negativo.

El efecto stroop se caracteriza por el incremento de la latencia cuando hay que nombrar el color de la tinta en el que una palabra está escrita, si ésta hace referencia a un color incongruente con el de la tinta (p.e. rojo escrito en verde), con respecto a una condición base en la que hay congruencia (p.e. verde escrito en verde). Este efecto proporciona evidencia de la dificultad para inhibir una respuesta sobreaprendida. Típicamente, el efecto Stroop es mayor en el caso de los pacientes comparado con los mayores sanos (Fisher, Freed y Corkin, 1990; Koss, Ober, Delis y Friedland, 1984). Koss et al. (1984) demuestran que este efecto sigue siendo mayor para los pacientes con EA incluso después de ajustar las puntuaciones de interferencia a la velocidad de procesamiento. Spieler, Balota y Faust (1996) y García-Viedma (2010) encuentran que los pacientes con EA cometen una mayor proporción de errores de intrusión cuando debían inhibir la respuesta automática de la lectura para indicar el color en los ensayos incongruentes. Bondi et al. (2002) confirman el efecto stroop con la puntuación de interferencia ajustada a la velocidad de procesamiento, y también encontraron que los pacientes cometen más errores de intrusión. Por su parte, Duchek et al. (2009) aplican una tarea stroop a un grupo de adultos jóvenes, un grupo de mayores y un grupo de pacientes con EA muy inicial y toman las medidas estándar de tiempo de reacción (TR) y de errores. También codifican la variabilidad individual ensayo a ensayo, para lo cual calculan el coeficiente de variación ( $\mathrm{CoV}$ ), que se computa dividiendo la desviación típica del sujeto por su media. Se ha propuesto que los cambios en esta medida a lo largo de los ensayos de una tarea es un indicador de las funciones neurocognitivas, y se señala que la variabilidad intra-tarea es consistente con una ruptura del sistema de control atencional o ejecutivo que permite mantener las metas de una tarea y controlar distintas alternativas de acción. Los resultados obtenidos con las medidas de tiempo de respuesta y errores replican los hallazgos de estudios previos, se observa un mayor efecto de congruencia en TR y más errores en los ensayos incongruentes en el caso de los pacientes con EA muy inicial que en los mayores sanos. De esta forma, se confirman las dificultades de los pacientes con EA inicial para controlar respuestas prepotentes. Al mismo tiempo, los resultados de variabilidad intra-individual reflejan un claro incremento de la misma en mayores y pacientes con EA, siendo superior en el caso de los pacientes. Por ello, Duchek et al. (2009) apun$\tan$ que el incremento de la variabilidad ensayo a ensayo en las fases iniciales de la EA es consistente con las dificultades de control. En un estudio posterior, Tse, Balota, Yap, Du- chek y McCabe (2010) examinan las características de las distribuciones de los TR en la tarea stroop. La lógica subyacente es que los incrementos simples en la variabilidad de los TR de los sujetos pueden deberse a cambios en todos los componentes exponenciales de la distribución de los TR, o a cambios en componentes específicos de la misma. Esta aproximación permite especificar si los cambios en la variabilidad intra-individual como una función de la edad o de la EA, se debe a un enlentecimiento general (reflejado por los cambios en todos los parámetros de la distribución) o no (reflejado por cambios en componentes específicos de la distribución). Pues bien, los resultados apuntan que en el caso de los mayores sanos se veían afectados todos los componentes de la distribución de los TR. Sin embargo, los pacientes con EA presentan más extenso el extremo de la distribución correspondiente a los valores más altos de TR que los mayores sanos. De tal forma que los cambios en los incrementos en la variabilidad intra-individual observados en su estudio previo (Duchek et al., 2009), se debían a que los pacientes tienen TR más altos en el extremo lento de la distribución. Es decir, los pacientes con EA leve necesitan más tiempo para evitar la información distractora.

La tarea Hayling valora la capacidad para suprimir respuestas verbales en un test de procesamiento semántico. Se presenta a los participantes una serie de oraciones cortas en las que la última palabra es omitida, pero se puede anticipar fácilmente. Los participantes deben escuchar cada oración y completarla, pero no con la palabra que se prevé, sino con una palabra no relacionada con la oración. De esta forma, esta tarea requiere la inhibición voluntaria de una respuesta automática. Collette, Van der Linden y Salmon (1999) no encuentran diferencias entre los pacientes con EA y los controles en la velocidad de respuesta, pero el grado de relación semántica de las respuestas dadas era considerablemente mayor en el caso de los pacientes, lo cual sugiere que tienen dificultades para inhibir respuestas irrelevantes pero relacionadas semánticamente con la tarea.

Otra tarea que permite valorar la interferencia a nivel de respuesta es la tarea Simon (1969). En una tarea Simon típica se presenta una palabra espacial (p.e. izquierda) en la misma (congruente) o distinta (incongruente) localización indicada por el significado de la palabra. A los participantes se les pide que indiquen el significado de la palabra e ignoren la localización de la misma. Con sujetos sanos típicamente se observa un incremento de la latencia de respuesta cuando el significado de la palabra y su localización son incongruentes, lo que refleja la dificultad para inhibir la respuesta prepotente asociada a la localización de la palabra cuando se intenta seleccionar el significado de ésta. Castel, Balota, Hutchison, Logan y Yap (2007) encuentran que tanto los adultos mayores sanos como los pacientes con EA muestran incrementos en la latencia de repuesta desproporcionados en la condición incongruente con respecto a los adultos jóvenes, siendo los sujetos con EA los que cometen más errores con respecto a los mayores sanos cuando hay conflicto entre el significado de la palabra y la localización de ésta. Del mismo modo, Fes- 
ta, Heindel y Ott (2010) aplican una tarea tipo Simon a un grupo de mayores sanos y a un grupo de pacientes con EA, y hayan un efecto de interferencia de respuesta Simon incrementado en los pacientes con EA con respecto a los mayores sanos. Por su parte, Duchek et al. (2009) también aplican una tarea Simon a un grupo de adultos jóvenes y mayores sanos y a un grupo de pacientes con EA muy inicial. Como ya se ha indicado en el apartado de la tarea stroop, examinan las diferencias de variabilidad intra-individual calculando el $\mathrm{CoV}$ y toman medidas estándar de TR y de errores. Los resultados con las medidas estándar replican hallazgos previos, observando cómo los pacientes con EA presen$\tan$ un mayor efecto de interferencia en la latencia de respuesta y más errores en los ensayos incongruentes que los mayores sanos. Del mismo modo, los resultados de variabilidad intra-individual reflejan un claro incremento de la misma en mayores y pacientes con EA, siendo superior en el caso de los pacientes. Al igual que ocurre en la tarea stroop, en un estudio posterior (Tse et al., 2010) se observa que los cambios en la variabilidad intra-individual como una función de la EA se deben únicamente a cambios en la cola lenta de la distribución de los TR, apoyando también la existencia de problemas de control ejecutivo.

Por su parte, la habilidad para suprimir movimientos oculares sacádicos intencionalmente se ha utilizado para valorar la inhibición motora de respuestas reflexivas (Muller y Rabbitt, 1989). La tarea antisacádica requiere inhibir un movimiento sacádico reflexivo dirigido hacia una clave inicial periférica (prosacádico), y en su lugar generar un movimiento sacádico en la dirección opuesta (antisacádico). La capacidad para controlar los movimientos sacádicos está más afectada en los pacientes con EA que en los mayores sanos. Los pacientes cometen más errores en los ensayos antisacádicos que los controles (Mulligan, Mackinnon, Jorm, Giannakopoulus y Michel, 1996), y la frecuencia de los errores prosacádicos en los ensayos antisacádico correlaciona con la severidad de la demencia (Currie, Ramsden, McArthur y Maruff, 1991).

Los test go-no go y stop signal son los principales paradigmas que se utilizan para explorar la inhibición de respuestas motoras. En el primero, se realiza una tarea de reacción de elecciones sucesivas que implican ensayos en los que tienen que responder a un estímulo target determinado, y ensayos en los que tienen que retener su respuesta a otros estímulos. Así, el paradigma go-no go implica la ejecución (ensayos go) e inhibición (ensayos no go) de una respuesta motora preparada. Con esta tarea se obtienen resultados contradictorios. Mientras Amieva et al. (2002) no encuentran evidencia de una inhibición deficiente de respuestas motoras preparadas, Collette, Van der Linden, Delrue y Salmon (2002) sí encuentran déficit en los pacientes con EA. Amieva et al. (2004) explican estas discrepancias indicando que, en ambos estudios, hay un $50 \%$ de ensayos "go" y un $50 \%$ de ensayos "no go", y que esto produciría un refuerzo relativamente débil de la respuesta motora en los ensayos "go". De esta forma, parece que las demandas de inhibición de respuesta no estarían muy claras en estas tareas. Estos autores apuntan que el incremento de la probabilidad de respuestas "go" en este paradigma, causaría más déficit en los pacientes con EA, ya que el aumento de la frecuencia de los ensayos "go" da lugar a una respuesta de preparación más débil en los pacientes con EA (Low y Miller, 1999), lo que hace que la supresión de la respuesta sea más difícil (Bruin y Wijers, 2002).

En el paradigma stop signal (Logan y Cowan, 1984), los participantes realizan una tarea de tiempo de reacción visual en la que deben interrumpir su respuesta en una serie de ocasiones en las que aparece, de manera infrecuente, una señal auditiva. Este paradigma proporciona una forma de valorar la habilidad de inhibir una respuesta dirigida por una clave externa. Amieva et al. (2002) observaron que los pacientes con EA cometían más errores (dar una respuesta aunque el tono indique que no se dé respuesta) que los controles, lo que sugiere dificultades para inhibir un plan motor predominante.

Todos estos estudios ponen de manifiesto que en los inicios de la EA se verían afectados aquellos procesos inhibitorios que requieren un procesamiento controlado. No obstante, también se han realizado estudios en los que no se han hallado dificultades relacionadas con la resolución del conflicto en los inicios de la EA. Fernández-Duque y Black (2006) aplicaron una versión modificada del Attentional Network Test (ANT) a personas mayores sanas y pacientes con EA. La ANT es una combinación de la tarea de atención encubierta de Posner (1980) y de la tarea de flancos de Eriksen y Eriksen (1974), que permite valorar las tres redes atencionales (Posner y Petersen, 1990). En la tarea de atención encubierta la atención es dirigida hacia una localización antes de que el target aparezca. La diferencia en tiempo de reacción (TR) entre ensayos en los que las claves son válidas (informan correctamente de la posición del target) e inválidas (no informan correctamente de la posición del target) constituye una medida de orientación. Otros ensayos incluyen claves que proporcionan información temporal pero no espacial sobre el inicio del target (clave neutral). Estos ensayos se comparan con ensayos en los que el target aparece sin una señal de aviso (no clave), y la diferencia en TR entre estos ensayos es una medida de alerta. Por su parte, en la tarea de flancos el estímulo objetivo se presenta junto a distractores con información congruente (el target y los distractores requieren responder con la mano izquierda, por ejemplo) o incongruente (el estímulo objetivo requiere responder con la derecha y los distractores con la izquierda). La diferencia en TR entre ensayos congruentes e incongruentes ofrece una medida de resolución de conflicto. Pues bien, FernándezDuque y Black (2006) combinaron en su versión de la ANT cuatro tipos de clave (válida, inválida, neutral y no-clave) con dos tipos de distractores (congruente e incongruente). Estos autores no encontraron problemas de resolución de conflictos en los pacientes con EA leve. Posteriormente, llevaron a cabo otro estudio (Fernández-Duque y Black, 2008) en el que utilizaron una tarea de TR en la que se enfatiza la velocidad para responder. Los sujetos debían responder a la di- 
rección de una flecha (de un color concreto) mientras ignoran los estímulos distractores (cualquier estímulo de otro color) (p.e. "si la flecha negra apunta a la derecha, presiona la tecla derecha"). El target podía aparecer sólo, con distractores neutrales o con una flecha distractora (en color distinto a la flecha objetivo). La comparación de los ensayos en los que aparecía el target sólo con los ensayos en los que aparecía éste junto a distractores neutrales, da una medida de filtrado perceptual. La comparación de los ensayos en los que el distractor y el target dan información congruente con los ensayos en los que el objetivo y los distractores ofrecen información incongruente, proporciona una medida de resolución de conflicto. De nuevo, no observaron diferencias entre los pacientes con EA y las personas mayores en la resolución de conflictos ni en el filtrado perceptual. Estos resultados llevaron a Fernández-Duque y Black a proponer que las dificultades encontradas en estudios previos se debían a problemas para mantener el set mental.

Se ha explorado también el control inhibitorio en la EA con respecto a otras patologías muy relacionadas con esta enfermedad, como es la demencia frontotemporal (DFT). Por ejemplo, Torralva, Roca, Gleichgerrcht, López y Manes (2009) comparan la ejecución en el test de screening INECO de un grupo de pacientes con EA, un grupo de pacientes con la variante conductual de la DFT y un grupo control. Este test incluye varias tareas relacionadas con el lóbulo frontal, entre las que se encuentra una tarea Hayling. Los resultados indican que hay diferencias significativas en la ejecución de los dos grupos de pacientes, siendo sensiblemente mejor la actuación de los EA que la de los pacientes con DFT. Parece, por tanto, que este test sobre control inhibitorio, ayudaría en el diagnóstico diferencial de estas dos entidades vinculadas al deterioro del funcionamiento ejecutivo. En esta misma línea, otro estudio más reciente, Hornberger et al. (2010) examina si el test Hayling permite discriminar entre ambos grupos de pacientes con demencia. Los resultados informan que el rendimiento de los pacientes con la variante conductual de la DFT es inferior al de los pacientes con EA. Este test permite discriminar entre ambos grupos de pacientes mejor que otros tests asociados al funcionamiento ejecutivo (p.e., tareas de fluidez fonética...). Los autores concluyen que las medidas de desinhibición parecen ser una poderosa herramienta diagnóstica para diferenciar entre ambos tipos de demencia.

En general, el análisis del control inhibitorio en mayores sanos con respecto a pacientes con EA leve, indica que estos últimos tienen déficit asociados a este componente de control; aunque los resultados de Fernández-Duque y Black (2006, 2008) plantean la posibilidad de que estas dificultades están vinculadas con problemas en el mantenimiento del set mental. Por otro lado, los estudios que exploran esta capacidad en los inicios de la EA con respecto a la DFT, informan que las alteraciones del control inhibitorio son menores en el caso de la EA; y señalan que este componente sería especialmente útil en el diagnóstico diferencial de ambos tipos de demencia.

\section{Coordinación de más de una tarea}

Este componente se ha valorado principalmente con el paradigma dual. En éste se realizan de forma concurrente dos tareas que implican recursos de procesamiento distintos, y se compara esta ejecución con realización de cada tarea por separado. Los sujetos normalmente invierten más tiempo en la realización conjunta de las tareas, en comparación con la realización aislada de cada una de ellas. Varios estudios han informado que los pacientes con EA inicial presentan déficit en tareas duales. La mayoría de estos estudios indican que los pacientes tienen una ejecución similar a los controles cuando las dos tareas se realizan por separado, pero muestran un declinar desproporcionado en la ejecución concurrente de ambas (Baddeley, Logie, Bressi, Della Sala y Spinnler, 1986; Collette, Van der Linden, Bechet y Salmon, 1999; Grady et al., 1989; Greene, Hodges y Baddeley, 1995; Grober y Sliwinski, 1991). Por ejemplo, Baddeley et al. (1986) utilizaron una tarea de seguimiento manual de un círculo en movimiento y otra de repetición de series de dígitos. Los autores observaron que la ejecución de la tarea de seguimiento sólo era deficiente en el caso de los pacientes con EA y que éstos mostraban un declive en su rendimiento en la situación dual. Utilizando una tarea similar, estudios más recientes también muestran un deterioro significativo de la actuación de los pacientes con EA en condiciones duales, mientras los mayores sanos tienen un rendimiento similar al de los jóvenes (Logie, Cocchini, Della Sala y Baddeley, 2004; MacPherson, Della Sala, Logie y Wilcock, 2007). Por otra parte, Baddeley, Bressi, Della Sala, Logie y Spinnler (1991) observaron en un estudio longitudinal, que el declive, conforme progresa la enfermedad, de la actuación de los pacientes con EA en la condición dual era mayor que en las tareas simples.

Además, Baddeley et al. (2001) han mostrado que el incremento de las demandas de las tareas no tiene mayor impacto en los pacientes que en los controles cuando se realizan separadamente, y que las deficiencias en las tareas duales pueden darse utilizando combinaciones de tareas distintas (por ejemplo, tareas de tachado y de repetición de secuencias aleatorias de dígitos, o de búsqueda visual y de detección auditiva). Los hallazgos apuntan que los pacientes con EA tienen dificultades específicas con esta capacidad y no con las demandas cognitivas generales. Della Sala y Logie (2001) y Logie et al. (2004) también han expuesto que estas dificultades son consistentes a lo largo de la práctica, que aparecen independientemente de las demandas cognitivas de cada tarea, y que puede darse cuando se combinan tareas que implican distintos recursos cognitivos. Por ejemplo, Rapp, Krampe y Baltes (2006) han encontrado que los pacientes con EA inicial presentaban dificultades en una tarea dual en la que combinan una tarea cognitiva y una tarea sensorio-motora de control postural.

Otro estudio más reciente es el de Festa et al. (2010). Aplican a un grupo de mayores sanos y a pacientes con EA 
diversas tareas como: a) Trazado: los sujetos deben mantener la posición de un coche dentro de una línea central en el monitor de la pantalla; b) Orientación: los sujetos responden ante la presencia de un estímulo. Aparece un punto de fijación con dos cuadrados a su derecha e izquierda. Tras un intervalo de tiempo variable, una clave (valida, no-válida o neutral) indica la posible localización del target, que aparecerá en uno de los dos cuadrados, y que podrá coincidir (clave válida) o no (clave no-válida) con la posición señalada por la clave; c) Simon: requiere a los participantes indicar la localización de una palabra mientras ignoran el significado de la misma (referente a una posición espacial; d) Búsqueda visual: los sujetos deben buscar y detectar la presencia de un estímulo previamente establecido (triángulo rojo) de entre una serie de distractores (triángulos azules, círculos rojos o cuadrados azules). A continuación combinaban la ejecución de la tarea de trazado con cada una de las tres restantes tareas. Las medidas utilizadas fueron TR y porcentaje de errores para las tres tareas, también se computaron la sensibilidad (d-prima) y el criterio de respuesta $(\beta)$ en la tarea de búsqueda visual. Los resultados confirman que los pacientes con EA presentan un déficit específico en la coordinación de distintas tareas, ya que la ejecución de todas las tareas se ve deteriorada en estas condiciones. Sin embargo, hay un dato llamativo. Las condiciones duales producían en el grupo de pacientes con EA, pero no en el de los mayores sanos, un cambio en la eficacia de las tareas de orientación, Simón y de búsqueda visual, que se correspondía con el siguiente patrón: la sensibilidad (d-prima) en la tarea de búsqueda visual disminuye, y el efecto de interferencia en las tareas de orientación espacial y Simon también disminuye. Parece que las condiciones duales atenúan los déficits observados en la tarea Simon y de orientación espacial cuando se realizan de forma aislada. No obstante, esto parece deberse, no a una mejor ejecución, que se reflejaría en una disminución de los costes provocados por información conflictiva (clave inválida en la tarea de orientación o respuesta incongruente en la tarea Simon), sino a una disminución de los beneficios que se derivan de la información congruente (en el caso de la tarea Simon) y de las claves válidas (en el caso de la tarea de orientación espacial).

En definitiva, todos estos estudios ponen de manifiesto que los déficit vinculados con la coordinación de más de una tarea son específicos de la EA, y que no aparecen durante el envejecimiento. También se ha investigado la especificidad de estos problemas en la EA con respecto a otros trastornos. Un ejemplo es un estudio reciente de Kaschel, Logie, Kazén y Della Sala (2009). En este trabajo examinaban la ejecución de una tarea dual en personas mayores sanas, personas mayores con depresión y pacientes con EA. Los sujetos realizan una tarea de recuerdo de dígitos presentados auditivamente y una tarea de trazado viso-espacial, por separado y de forma conjunta. Para valorar el rendimiento de los participantes en la condición dual ajustaban las demandas de las tareas simples a la capacidad de cada individuo. Los resultados mostraron que el grupo de EA era diferencialmente sensible a la condición dual con respecto a los otros dos grupos. De tal forma que los costos asociados a la realización concurrente de las dos tareas eran específicos de los pacientes con EA, y los mayores sanos y los mayores con depresión tenían una ejecución similar. Además, las dificultades asociadas a las tareas duales de los pacientes con EA con respecto a los mayores con depresión, se mantenían incluso cuando ambos grupos eran igualados en su rendimiento en tareas de memoria episódica. Por tanto, estos hallazgos refuerzan la idea de que los problemas relacionados con la coordinación de más de una tarea son específicos de la EA.

\section{Cambio de configuración mental}

Como ya se ha apuntado, este componente se ha explorado clásicamente con el paradigma "task-switching" o cambio de tarea, en el cual los participantes realizan la misma tarea o alternan entre diferentes tareas. Un hallazgo consistente en este paradigma es que los sujetos necesitan más tiempo para responder cuando tienen que cambiar de tarea que cuando repiten la misma tarea, y a este incremento en el tiempo de respuesta se le conoce como costos de cambio. Así, la evidencia de los procesos de control en esta tarea es proporcionada por los costos de cambio globales (expresados por un incremento en el tiempo de reacción durante la realización de un bloque de ensayos en el que se alternan tareas, con respecto a un bloque de ensayos en el que se realiza la misma tarea), y por los costos de cambio específicos (reflejados en el incremento del tiempo de respuesta en el primer ensayo de una nueva tarea con respecto a los ensayos que no implican cambio de tarea). Los costos de cambio globales reflejarían la dificultad asociada al mantenimiento y programación de varias configuraciones mentales; mientras que los costos de cambio específicos informarían de las demandas de los procesos ejecutivos asociados al cambio actual (Verhaeghen y Basak, 2005; Verhaeghen, Cerella, Bopp y Basak, 2005).

Belleville, Bherer, Lepage, Chertkow y Gauthier (2008) llevan a cabo un experimento sobre la capacidad de cambio en pacientes con EA leve, personas con deterioro cognitivo leve (DCL) y mayores sanos. Ellos diseñan dos tareas basadas en el paradigma de cambio de tarea, en las que se presenta una serie de dígitos binarios. En la versión espacial, los sujetos tienen que leer el dígito que se encuentra a la derecha del punto de fijación en unas ocasiones, y a la izquierda en otras. En la versión conceptual los sujetos tienen que sumar los dígitos en unas ocasiones y restarlos en otras. El cambio de una tarea a otra en ambas versiones era indicado por una clave que se presenta de forma aleatoria. Utilizan como medidas los costos de cambio globales y los costos de cambio específicos Los resultados muestran que los pacientes tienen mayores costos de cambio globales y específicos que los mayores sanos, y que estos déficit se limitan a la versión espacial. Los autores concluyen que los pacientes con EA leve presentan problemas relacionados con el mantenimiento y 
reconfiguración de varias operaciones mentales, que no mejoraban con la práctica.

Fernández-Duque y Black (2008) examinan si el envejecimiento y los inicios de la EA se acompañan de un incremento en los costos de cambio globales. Los sujetos deben responder a la dirección de una flecha (de un color concreto) mientras ignoran los estímulos distractores (flecha de otro color) (p.e. "si la flecha blanca apunta a la izquierda, presiona la tecla izquierda"). En este experimento el color del target cambia cada cuatro ensayos y una clave recuerda a los sujetos el color del estímulo objetivo al comienzo de la serie de cuatro ensayos (p. e. la palabra "negro" en color "negro"). En un primer bloque de ensayos sólo presentan flechas y la respuesta de los participantes es determinada por el propio estímulo, puesto que no hay distractores y el color de la flecha indica a qué dirección responder. En un segundo bloque, dos flechas concéntricas (una blanca y otra negra) se presentan de forma simultánea, y éstas podían apuntar en la misma dirección o en dirección opuesta. El color determina cuál era la flecha clave, y éste cambia de nuevo cada cuatro ensayos. Los resultados revelan que los costos de cambio globales son mayores en los pacientes con EA leve que en las personas mayores. Los autores concluyen que las dificultades de los pacientes con EA leve se relacionan con una incapacidad para mantener un set mental alternante en presencia de información distractora.

García-Viedma (2010) también ha explorado esta capacidad en pacientes con EA inicial utilizando este paradigma. La tarea consiste en solucionar una serie de operaciones aritméticas muy sencillas (sumar 1 o restar 1 a dígitos simples), de tal forma que en unos bloques o se suma o se resta, mientras que en otros, las sumas y las restas se alternan. Además, consta de dos condiciones: una en la que se presenta la operación aritmética a resolver en cada ensayo (p.e. 9$1=)$, y otra en la que el participante debe sumar 1 o restar 1 al dígito que se presenta (p.e. 8) en función de la orden presentada previamente (SUMA 1 o RESTA 1). Se toman como medidas los costos de cambio globales y los costos de cambio específicos, así como la latencia de respuesta y los errores. Los resultados ponen de manifiesto, por un lado, que los pacientes presentan mayores costos de cambio globales, y unos tiempos de respuesta y porcentaje de errores superiores a los del grupo de mayores sanos. Estos mayores costos globales apuntan hacia dificultades relacionadas con el mantenimiento y configuración de distintas operaciones mentales o programas en los inicios de la EA. Asimismo, los resultados ponen de manifiesto que los déficit mostrados por los pacientes no pueden explicarse por una disipación del set, sino más bien, estos déficit parecen deberse a un efecto de interferencia entre set diversos que impediría la configuración apropiada de distintas operaciones mentales. Por otro lado, cuando se compara la ejecución de ambos grupos en función de la condición, se observan mayores tiempos de respuesta en el grupo de pacientes con respecto al grupo control para ambas condiciones, siendo éstos mayores en el caso de la condición en la que se presenta la operación. En la base de los resultados parece estar un posible efecto de preparación, por el cual, cuando se da previamente la orden el sujeto puede anticipar su respuesta y los tiempos de reacción disminuyen.

Por su parte, Duchek et al. (2009) también han explorado las diferencias de variabilidad intra-individual (utilizando como medida el $\mathrm{CoV}$ ) en una tarea de cambio en un grupo de adultos jóvenes, un grupo de mayores y un grupo de pacientes con EA muy inicial. Además tomaron medidas estándar de tiempo de reacción y de errores, al igual que hicieron con la tarea stroop y la tarea Simon (véase descripción más detallada de las medidas y su justificación en el apartado Control inhibitorio). En esta tarea los participantes tienen que realizar una actividad distinta cada dos ensayos. En cada ensayo aparecen un par de estímulos (una letra y un número) en el centro de la pantalla, y encima una clave que indica la tarea que tienen que realizar: "letra" (VC) o "número" (PI). En los ensayos "letra" deben indicar si la letra es vocal o consonante (VC), y en los ensayos "número" si éste es par o impar (PI). Los participantes presionan la tecla $d$ si la letra era consonante o el número impar, y la tecla $k$ si la letra era vocal o el número consonante. Los resultados indican que los pacientes presentan costos de cambio locales para los TR más pequeños y más costos generales para los errores, que los mayores y jóvenes sanos. Los autores señalan que los pacientes estaban menos "sintonizados" con las restricciones de la tarea, y por lo tanto su ejecución se veía menos afectada por los cambios entre los ensayos, lo cual sugiere una alteración del sistema de control ejecutivo que mantiene las metas de la tarea. Al igual que ocurriera en la tarea stroop y la tarea Simon, los datos de variabilidad intraindividual ponen de manifiesto que ésta era mayor en el caso de los pacientes. De nuevo se apunta que el incremento de la variabilidad ensayo a ensayo en las fases iniciales de la EA es consistente con las dificultades de control ejecutivo. En un estudio posterior, Tse et al. (2010), utilizando esta tarea, encontraron que los cambios en la variabilidad intra-individual en función de la EA, también se debe a la presencia de valores de TR mucho más altos en la cola de la distribución correspondiente a los TR más lentos, confirmando así la existencia de problemas de control atencional.

Esta operación de control también se ha explorado cualitativamente a través de la ejecución en tests tradicionales. Aunque existen pocos estudios de este tipo, los resultados obtenidos por los pacientes con EA indican la presencia de problemas con la capacidad de cambio. Por ejemplo, Bondi, Monsch, Butters y Salmon (1993) encuentran que los pacientes con EA tienen más problemas para descubrir nuevas reglas en el Wisconsin Card Sorting Test (WCST) (Heaton, 1981), y que cometen más errores perseverativos que los controles en este test. Estas dificultades también se han observado en una versión del WCST, el Modified Card Sorting Test (MCST) (Nelson, 1976), obteniendo los pacientes con EA una peor ejecución que los controles (Calderon et al., 2001; Traykov et al, 2002). Por otra parte, los pacientes con 
EA muestran problemas con la atención alternante en el Trail Making Test (García-Viedma, 2010; Reitan, 1958).

Todos estos resultados parecen confirmar que los pacientes con EA leve muestran dificultades relacionadas con la capacidad para mantener y configurar distintas set mentales.

\section{Actualización de la memoria de trabajo}

Como previamente se ha indicado, esta operación de control puede explorarse utilizando el paradigma "running memory", las tareas " $n$-back", y las tareas de amplitud de memoria de trabajo. García-Viedma (2010) valora esta capacidad utilizando una tarea de amplitud de memoria de trabajo. Las tareas de amplitud de memoria de trabajo, en su forma más típica, implican presentar una serie de oraciones que deben ser procesadas, y almacenar la última palabra de cada una de ellas. Al final de la secuencia de frases se pide que recuerden la última palabra de cada una. El número máximo de palabras que puede mantenerse mientras se procesan las oraciones, se corresponde con la amplitud de memoria de trabajo verbal del sujeto. García-Viedma (2010) aplicó un subtest de la batería ECCO (López-Higes, del Río y Fernández-Guinea, 2005) que es una adaptación de la tarea de Daneman y Carpenter (1980). Esta tarea consiste en la presentación escrita de grupos de oraciones que contienen de 2 a 6 frases. El participante tiene que leer las oraciones en voz alta y mantener en la memoria la última palabra de cada oración. Tras la presentación de todas las oraciones del grupo, el participante debe decir todas las palabras últimas que recuerda. El número de oraciones de los grupos se va aumentando a medida que avanza la tarea, de manera que también se aumenta la carga de la memoria de trabajo. Los resultados informan que los pacientes con EA obtienen una puntuación inferior en esta tarea con respecto al grupo control.

Otro estudio es el de Rochon, Waters y Caplan (2000), en el que valoran esta capacidad en pacientes con EA en un estadio leve a moderado. La tarea, denominada tarea de amplitud de memoria de trabajo de puntos, consiste en presentar a los participantes cartas con nueve puntos de diferentes colores. La tarea de los sujetos es contar el número de puntos azules que se encuentran en cada carta, que puede ir de 1 a 9, y mantenerlo en la memoria hasta el final de una secuencia. Al finalizar cada secuencia, se pide que indique de forma serial el número de puntos azules que tenían las cartas presentadas. En este estudio también se observa una peor ejecución en el caso de los pacientes con EA.

\section{Conclusiones}

Los resultados muestran que los pacientes con EA leve presentarían dificultades en cada una de las operaciones de control atencional. De este modo, en fases iniciales se encontrarían problemas relacionados con la inhibición de información irrelevante, la coordinación de la realización concurrente de más de una tarea, el cambio de configuración mental, y la actualización de la memoria de trabajo. Por tanto, se pone de manifiesto que en los inicios de la EA se observa un deterioro generalizado del CE.

El conocimiento que nos aportan los estudios sobre la afectación del CE en las primeras etapas de la enfermedad de Alzheimer es muy relevante, tanto a nivel teórico como clínico o aplicado. No cabe duda que el poder relacionar la afectación gradual de los componentes del CE con el proceso de desarrollo neuropatológico de la EA, abre un conjunto de posibilidades que merecen ser analizadas, tanto en su relación con el diagnóstico temprano y diferencial de este proceso neurodegenerativo, como con el estudio de los correlatos anatomofisiológicos de CE como proceso cognitivo.

Los hallazgos científicos apuntan a la afectación temprana del CE en los pacientes con EA, por lo que se debe considerar su papel como marcador neuropsicológico de la misma, y analizar su implicación en los individuos con deterioro cognitivo leve que se convierten a enfermos de Alzheimer. La detección temprana de estos casos es muy necesaria hoy en día, especialmente si se tienen en cuenta los beneficios que en las primeras fases tienen los tratamientos farmacológicos y las intervenciones neuropsicológicas. También se debe considerar la importancia del diagnóstico diferencial con otras patologías similares, y los datos que se han recogido en esta revisión, indican cómo la diferente actuación en las pruebas de CE distinguen, por ejemplo, a los pacientes con EA de los que padecen una demencia fronto-temporal o al mismo proceso de envejecimiento normal.

La aportación de los estudios de neuroimagen es muy apreciable en relación con el substrato neuronal del CE, y las complejas interconexiones que se establecen entre redes y áreas cerebrales. Y del mismo modo, para comprender la neuropatología progresiva de la EA, y sus síntomas clínicos, cognitivos y conductuales. En este sentido, es imprescindible incluir en este análisis las evidencias que ofrecen el rendimiento en las pruebas y tests neuropsicológicos que valoran los distintos subcomponentes del CE.

Desde un punto de vista más aplicado, el conocimiento de la afectación temprana del CE en los pacientes con EA, permite explicar las distintas manifestaciones clínicas y la alteración de diversos dominios cognitivos y conductuales (Alberoni, et al., 1992; Amieva et al., 2004; Belleville et al., 2003; Collette y Van der Linden, 2002; Duchek et al., 1998; Martini et al., 2003; Perry y Hodges, 1999). Los trastornos de los subcomponentes del CE implican dificultades con la manipulación activa de la información, que son necesarias en la realización de actividades complejas o nuevas. Estas operaciones cognitivas tienen un papel relevante en numerosas situaciones de la vida diaria. Actividades como cocinar, hablar mientras se camina, conducir, comprobar el cambio en el supermercado o mantener conversaciones..., requieren evitar que la información relevante interfiera en las mismas, alternar entre distintas fuentes de información o estímulos o tareas, realizar de forma simultánea distintas acciones... Precisamente los problemas para llevar a cabo estas actividades constituyen las primeras quejas de las personas con EA y de sus familiares.

No cabe duda que este conocimiento más detallado de los subcomponentes del EC y de su afectación, abre el campo para el desarrollo de tests y tareas neuropsicológicas con un enfoque más ecológico y sensible para detectar los primeros problemas 
que presentan las personas que sufren este tipo de enfermedades. Se deberían, por tanto, incluir en los protocolos de evaluación y detección de las demencias. Del mismo modo, los programas de estimulación e intervención neuropsicológica pueden recoger esta información tan importante para diseñar programas, métodos y técnicas que se ajusten de manera más indivi-

\section{Referencias}

Alberoni, M., Baddeley, A. D., Della Sala, S., Logie, R. H. y Spinnler, H. (1992). Keeping track of a conversation: impairments in Alzheimer's disease. Int J Geriatr Psychiatry, 7, 639-646. doi: 10.1002/gps.930070905

Allport, A., Styles, E. A. y Hsieh, S. (1994). Shifting intentional set: Exploring the dynamic control of tasks. In C. Umiltá y M. Moscovitch (Eds.) Attention and Performance IV. (pp. 421-452). Cambridge, MA: MIT Press.

Amieva, H., Lafont, S., Auriacombe, S., Le Carret, N., Dartigues, J. F., Orgogozo, J. M. y Fabrigoule, C. (2002). Inhibitory breakdown and dementia of the Alzheimer type: a general phenomenon? J Clin Exp Neuropsychol, 24, 503-516. doi: 10.1076/jcen.24.4.503.1034

Amieva, H., Phillips, K. H., Della Sala, S. y Henry, J. D. (2004). Inhibitory functioning in Alzheimer's disease. Brain, 127, 949-964. doi: 10.1093/brain/awh045

Azari, N. P., Rapoport, S. I., Grady, C. L., Schapiro, M. B., Salerno, J. A., Gonzales-Aviles, A. y Horwitz, B. (1992). Patterns of interregional correlations of cerebral glucose metabolic rates in patients with dementia of the Alzheimer type. Neurodegeneration, 1, 101-111. Retrieved from http://www.sciencedirect.com/science/journal/10558330

Baddeley, A. D. (1996). Exploring the Central Executive. Q J Exp Psychol, 49A(1), 5-28. doi:10.1080/027249896392784

Baddeley, A. D., Baddeley, H., Bucks, R. S. y Wilcox, G. K. (2001). Attentional control in Alzheimer`s disease. Brain, 124, 1492-1508, doi:10.1093/brain/124.8.1492

Baddeley, A. D, Bressi, S., Della Sala, S., Logie, R. y Spinnler, H. (1991). The decline of working memory in Alzheimer's disease: a longitudinal study. Brain, 114, 2521-2542. doi:10.1093/brain/114.6.2521

Baddeley, A. D., Logie, R., Bressi, S., Della Sala, S. y Spinnler, H. (1986). Dementia and working memory. Q J Exp Psychol, 38A, 603-618. doi: 10.1080/14640748608401616

Belleville, S., Bherer, L., Lepage, E., Chertkow, H. y Gauthier, S. (2008). Task switching capacities in persons with Alzheimer's disease and mild cognitive impairment. Neuropsychologia, 46(8), 2225-33. doi: 10.1016/j.neuropsychologia.2008.02.012

Belleville, S., Rouleau, N. y Van der Linden, M. (2003). Effect of Manipulation and Irrelevant noise on Working Memory Capacity of Patients with Alzheimer's Dementia. Neuropsychology, 17(1), 69-81. doi:10.1037/08944105.17.1.69

Berendse, H. W., Verbundt, J. P. A, Scheltens, P. H., van Dijk, B. W. y Jonkman, E. J. (2000). Magnetoencephalography analysis of cortical activity in Alzheimer's disease: A pilot study. Clin Neurophisiol, 11, 604-612. doi:10.1016/S1388-2457(99)00309-0

Bondi, M. W., Monsch, A. U., Butters, N. y Salmon, D. P. (1993). Utility of a modified version of the Wisconsin Card Sorting test in the detection of dementia of the Alzheimer type. Clinical Neuropsychologist, 7(2), 161170. doi:10.1080/13854049308401518

Bondi, M. W., Serody, A. B., Chan, A. S., Eberson-Shumate, S. C., Delis, D. C., Hansen, L. A. y Salmon, D. P. (2002). Cognitive and neuropathologic correlates of Stroop Color-Word Test performance in Alzheimer's disease. Neuropsychology, 16, 335-343. doi:10.1037//08944105.16.3.335

Botvinick, M. M., Braver, T. S., Carter, C. S., Barch, D. M., y Cohen, J. D. (2001). Conflict monitoring and cognitive control. Psychological Review, 108, 624-652. doi: 10.1037//0033-295X.108.3.624

Brown, R. E. y Marsden, C. D. (1988). Subcortical dementia: the neuropsychological evidence. Neuroscience, 25, 363-387. doi:10.1016/03064522(88)90246-1

Bruin, K. J. y Wijers, A. A. (2002). Inhibition, response mode, and stimulus probability: a comparative event-related potential study. Clin Neurophysiol, 113, 1172-1182. doi:10.1016/S1388-2457(02)00141-4 dual a las características de cada persona, y que permitan aminorar los síntomas de la enfermedad, potenciar las habilidades y capacidades en cada fase de su evolución, y retrasar, en la medida de los posible, el declive cognitivo y conductual de estas personas.

Bunge, S. A., Klinberg, T., Jacobse, R. B. y Gabrieli, J. D. E. (2000). A resource model of the neural basis of executive working memory. Proc Natl Acad Sci US A, 97, 3573-3578. doi:10.1073/pnas.050583797

Burgess, P. W. y Shallice, T. (1996). Response suppression, initiation and strategy use following frontal lobe lesions. Neuropsychologia, 34, 263-272. doi:10.1016/0028-3932(95)00104-2

Calderon, J., Perry, R. J., Erzinclioglu, S. W., Berrios, G. E., Dening, T. R. y Hodges, J. R. (2001). Perception, attention, and working memory are disproportionately impaired in dementia with Lewy bodies compared with Alzheimer's disease, I Neurol Neurosurg Psychiatry, 70, 157-164. doi:10.1136/jnnp.70.2.157

Castel, A. D., Balota, D. A., Hutchison, K. A., Logan, J. M. y Yap, M. J (2007). Spatial attention and response control in healthy younger and older adults and individuals with Alzheimer's disease: Evidence for disproportionate selection impairments in the Simon task. Neuropsychology, 21, 170-182. doi: 10.1037/0894-4105.21.2.170

Collette, M. y Van der Linden, M. (2002). Brain imaging oh the central executive component of working memory. Neurosci Biobehav Rev, 26, 105 125. doi:10.1016/S0149-7634(01)00063-X

Collette, F., Van der Linden, M., Bechet, S. y Salmon, E. (1999). Phonological loop and central executive functioning in Alzheimer's disease. Neuropsychologia, 37, 905-918. doi:10.1016/S0028-3932(98)00148-1

Collette, F., Van der Linden, M., Delrue, G. y Salmon, E. (2002). Frontal hypometabolism does not explain inhibitory dysfunction in Alzheimer's disease. Alzheimer Dis Assoc Disord, 16, 228-238. Retrieved from http://journals.lww.com/alzheimerjournal/pages/default.aspx

Collette, F., Van der Linden, M. y Salmon, E. (1999). Executive dysfunction in Alzheimer's disease. Cortex, 35, 57-72. doi:10.1016/S00109452(08)70785-8

Cummings, J. L. (1993). Frontal-subcortical circuits and human behavior. Arch Neurol, 50, 873-880. Retrieved from http://archneur.amaassn.org/

Currie, J., Ramsden, B., McArthur, C. y Maruff, P. (1991). Validation of a clinical antisaccadic eye movement test in the assessment of dementia. Arch Neurol, 48, 644-648. Retrieved from http://archneur.amaassn.org/

Damasio A. R. (1998). The somatic marker hypothesis and the possible functions of the prefrontal cortex. In Roberts AC, Robbins TW, Weiskrantz L, (Eds.). The frontal cortex: executive and cognitive functions. New York: Oxford University Press.

Daneman, M. y Carpenter, P. A. (1980). Individual differences in working memory and reading. Journal of Verbal Learning and Verbal Behavior, 19 450-466. doi:10.1016/S0022-5371(80)90312-6

Delbeuck, X., Collette, F. y Van der Linden, M. (2007). Alzheimer's disease as a disconnection syndrome? Neuropsychologia, 45(14), 33315-33323. doi:10.1016/j.neuropsychologia.2007.05.001

Della Sala, S. y Logie, R. H. (2001). Theoretical and Practical Implications of Dual-task Performance in Alzheimer's disease. Brain, 124, 1479-1481. doi:10.1093/brain/124.8.1479

Duchek, J. M., Balota, D. A., Tse, C. S., Holtzman, D. M., Fagan, A. M., Goate, A. M. (2009). The Utility of Intraindividual Variability in Selective Attention Tasks as an Early Marker for Alzheimer's Disease. Neuropsychology, 23, 746-758. doi: 10.1037/a0016583

Duchek, J. M., Hunt, L. A., Ball, K., Buckles, V. y Morris, J. C. (1998). Attention and driving performance in Alzheimer's disease. J Gerontol, 53B, 130-141. doi:10.1093/geronb/53B.2.P130

D’Esposito, M., Detre, J. A., Alsop, C. D., Shin, R. K., Atlas, S. y Grossman, M. (1995). The neural basis of the central executive of working memory. Nature, 378, 279-281. doi:10.1038/378279a0 
Eriksen, B. A. y Eriksen, C. W. (1974). Effects of noise letters upon the identification of a target letter in a nonsearch task. Perception \& Psychophysics, 16, 143-149. Retrieved from http://www.springerlink.com/content/1943-3921/16/1/

Estévez-González, A., García-Sánchez, C. y Junqué, C. (1997). La atención: una compleja función cerebral. Rev Neurol, 25(148), 1989-1997. Retrieved from http://www.revneurol.com/

Faglioni, P. (1999). The frontal lobe. In G. Denes y L. Pizzamiglio (Eds.) Handbook of clinical and experimental neuropsychology. East Sussex: Psychology Press, Taylor y Francis.

Faust, M. E., Balota, D. A., Duchek, J. M., Gernsbacher, M. A. y Smith, S. (1997). Inhibitory control during sentence comprehension in individuals with dementia of the Alzheimer type. Brain \& Language, 57, 225-253. doi:10.1006/brln.1997.1747

Fernandez-Duque, D. y Black, S. E. (2006). Attentional networks in normal aging and Alzheimer's Disease. Neuropsychology, 20(2), 133-143. doi: 10.1037/0894-4105.20.2.133

Fernandez-Duque, D. y Black, S. (2008). Selective attention in early dementia of Alzheimer type. Brain \& Cognition, 66(3), 221-231. doi:10.1016/j.bandc.2007.08.003

Festa E. K., Heindel W. C. y Ott B. R. (2010). Dual-task conditions modulate the efficiency of selective attention mechanisms in Alzheimer's disease. Neuropsychologia, 48, 3252-3261. doi:10.1016/j.neuropsychologia.2010.07.003

Fisher, L. M., Freed, D. M. y Corkin, S. (1990). Stroop Color-Word Test performance in patients with Alzheimer's disease. J Clin Exp Neuropsychol, 12, 745-758. doi: 10.1080/01688639008401016

Fontaine, A. Azouvi, P., Remy, P., Bussel, B. y Samson, Y. (1999). Functional anatomy of neurospychological deficits after TBI. Neurology, 53 (9), 1963-1968. Retrieved from http://www.neurology.org/content/53/9/1963.long

Foong, J., Rozewicz, L., Quaghebeur, G., Davie, C. A. y Kartsounis, L. D. (1997). Exenocutive function in multiple sclerosis. The role of frontal lobe pathology. Brain, 120, 15-26. doi:10.1093/brain/120.1.15

Fuster, J.M. (1997). The prefrontal cortex: anatomy, physiology and neuropsychology of the frontal lobe. New York: Lippincott-Raven.

Fuster J. M. (2002). Physiology of executive functions: the perception action cycle. In Stuss DT, Knight RT (Eds.). Principles of frontal lobe function (pp. 96-108). New York: Oxford University Press.

García-Viedma, M. R. (2010). Valoración del control atencional como marcador cognitivo del inicio de la enfermedad de Alz̧heimer. Granada: Colección Tesis Doctoral.

Goldman-Rakic, P. S. (1988). Topography of Cognition: Parallel distributed networks in primate association cortex. Annu Rev Neurosci, 11, 137-156. doi:10.1146/annurev.neuro.11.1.137

Grady, C. L., Furey, M. L., Pietrini, P., Horwitz, B. y Rapoport, S. I. (2001). Altered brain functional connectivity and impaired short-term memory in Alzheimer's disease. Brain, 124, 739-756. doi:10.1093/brain/124.4.739

Grady, C. L., Grimes, A. M., Patronas, N., Sunderland, T., Foster, N. L. y Rapoport, S. I. (1989). Divided attention, as measured by dichotic speech performance, in dementia of the Alzheimer type. Arch Neurol, 46, 317-320. Retrieved from http://archneur.ama-assn.org/

Greene, J. D. W., Hodges, J. R. y Baddeley, A. D. (1995). Autobiographical memory and executive function in early dementia of Alzheimer type. Neuropsychologia, 33, 1647-1670. doi:10.1016/0028-3932(95)00046-1

Grober, E. y Sliwinski, M. J. (1991). Dual-task performance in demented and nondemented elderly. J Clin Exp Neuropsychol, 13, 667-676. doi: 10.1080/01688639108401081

Heaton, R.K. (1981). The Wisconsin Card Sorting Test manual. Odessa: Psychological Assesment Resources.

Hornberger, M., Savage, S., Hsieh, S., Mioshi, E. Piguet, O. y Hodges, J. R. (2010). Orbitofrontal dysfunction discriminates behavioral variant of Frontotemporal dementia from Alzheimer's disease. Dement Geriatr Cogn Disord, 30, 547-552. doi:10.1159/000321670

Horwitz, B., Grady, C. L., Sclageter, N. L., Duara, R. y Rapoport, S. I. (1987). Intercorrelations of regional glucose metabolic rates in Alzheimer's disease. Brain Res, 407, 294-306. doi:10.1016/00068993(87)91107-3
Houghton, G. y Tipper, S. P. (1994). A model of inhibitory mechanisms in selective attention. In D. Dagenbach y T. H. Carr (Eds.). Inbibitory processes in attention, memory, and language. (pp. 53-112). San Diego (CA): Academic Press.

Jonides, J., Schumacher, E. H., Smith, E. E., Lauber, E. J., Awh, E., Minoshima, S. y Koeppe, R. A. (1997). Verbal working memory load affects regional brain activation as measured by PET. J Cogn Neurosci, 9, 462475. doi:10.1162/jocn.1997.9.4.462

Karatekin, C., Lazareff, J. A. y Asarnow, R. F. (2000). Relevance of the cerebellar hemispheres for executive functions. Pediatric Neurology, 22, 106112. doi:10.1016/S0887-8994(99)00128-9

Kaschel, R., Logie, R. H., Kazén, M. y Della Sala, S. (2009). Alzheimer's Disease, but not ageing or chronic depression, affects dual-tasking. J Neurol, 256, 1860-1868. doi:10.1007/s00415-009-5210-7

Kiesel, A., Steinhauser, M., Wendt, M., Falkenstein, M., Jost, K., Philipp, A. M., y Koch, I. (2010). Control and interference in task switching - A review. Psychol Bull, 136, 849-874. doi:10.1037/a0019842

Koechlin, E., Ody, C. y Kouneiher, F. (2003). The architecture of cognitive control in the human prefrontal cortex. Science, 302, 1181-1185. doi: 10.1126 / science. 1088545

Konishi, S., Nakajima, K., Uchida, I., Kameyama, M., Nakahara, K., Sekihara, K. y Miyashita, Y. (1998). Transient activation of inferior prefrontal cortex during cognitive set shifting. Nature Neurosci, 1, 80-84. doi:10.1038/283

Koss, E., Ober, B. A., Delis, D. C. y Friedland, R. P. (1984). The Stroop Color-Word Test: indicator of dementia severity. Int J Neurosci, 24, 5361. doi:10.3109/00207458409079534

Langley, L. K., Overmier, J. B., Knopman, D. S. y Prod'homme, M. M. (1998). Inhibition and habituation: preserved mechanisms of attentional selection in aging and Alzheimer's disease. Neuropsychol, 12, 353-366. doi:10.1037/0894-4105.12.3.353

Leskela, M., Heitanen, M., Kalska, H., Ylikoski, R. y Pdijasvaara, T. (1999). Executive functions and speed of mental procesing in elderly patients with frontal or no frontal ischemic stroke. Eur J Neurol, 6, 653-661. doi:10.1046/j.1468-1331.1999.660653.x

Leung, H. C., Skudlarski, P., Gatenby, J. C., Peterson, B. S. y Gore, J. C. (2000). An event-related functional MRI study of the stroop color word interference task. Cereb Cortex, 10, 552-560. doi:10.1093/cercor/10.6.552

Logan, G. D. y Cowan, W. (1984). On the ability to inhibit thought and action: a theory of an act of control. Psychol Rev, 91, 295-327. doi:10.1037//0033-295X.91.3.295

Logan, G. D. (2003). Executive control of thought and action: In search of the wild homunculus. Curr Dir Psychol Sci, 12, 45-48. doi: 10.1111/14678721.01223

Logie, R. H., Cocchini, G., Della Sala, S. y Baddeley, A. D. (2004). Is there a specific executive capacity for dual task co-ordination? Evidence from Alzheimer's Disease. Neuropsychology, 18, 504-513. doi: 10.1037/08944105.18.3.504

López-Higes, R. S., Del Río, D. y Fernández Guinea, S. (2005). Batería ECCO: Exploración Cognitiva de la Comprensión de Oraciones. Madrid: Editorial EOS.

Low, K. A. y Miller, J. (1999). The usefulness of partial information: effects of go probability in the choice/nogo task. Psychophysiology, 36, 288-297. doi:10.1017/S0048577299980332

Martini, L., Benke, T., Domahs, F. y Delazer, M. (2003). Everyday numerical abilities in Alzheimer's disease. J Int Neuropsychol Soc, 9, 871-878. doi:10.1017/S1355617703960073

MacPherson, S. E., Della Sala, S., Logie, R. H. y Wilcock, G. K. (2007). Specific AD Impairment in Concurrent Performance of Two Memory Tasks. Cortex, 43, 858-865. Retrieved from http://www.psy.ed.ac.uk/people/rlogie/142.pdf

Miller, E. K. y Cohen, J. D. (2001). An integrative theory of frontal lobe function. Annu Rev Neurosci, 24, 167-202. doi: 10.1146/annurev.neuro.24.1.167

Miyake, A., Friedman, N. P., Emerson, M. I., Witzki, A. H., Howerter, A. y Wager, T. D. (2000). The unity and diversity of executive functions and their contributions to complex "Frontal Lobe" task: A latent variable analysis. Cognit Psychol, 41, 49-100. doi:10.1006/cogp.1999.0734 
Monsell, S. (2003). Task-switching. Trends Cogn Sci, 7, 134-140. doi:10.1016/S1364-6613(03)00028-7

Monsell, S. y Mizon, G. A. (2006). Can the task-cuing paradigm measure an endogenous task-set reconfiguration process? J Exp Psychol Hum Percept Perform, 32, 493-516. doi:10.1037/0096-1523.32.3.493

Muller, H. J. y Rabbitt, P. M. (1989). Reflexive and voluntary orienting of visual attention: time course of activation and resistance to interruption. J Exp Psychol Hum Percept Perform, 15, 315-330. doi:10.1037/00961523.15.2.315

Mulligan, R., Mackinnon, A., Jorm, A. F., Giannakopoulos, P. y Michel, J. P. (1996). A comparison of alternative methods of screening for dementia in clinical settings. Arch Neurol, 53, 532-536. Retrieved from http://archneur.ama-assn.org/

Neill, W. T., Valdes, L. A. y Terry, K. M. (1995). Selective attention and the inhibitory control of cognition. In F. N. Dempster y C. J. Brainerd (Eds.). Interference and inbibition in cognition. (207-261). San Diego (CA): Academic Press.

Nelson, H. E. (1976). A modified Card Sorting Test sensitive to frontal lobe defects. Cortex, 12, 313-324. Retrieved from http://www.sciencedirect.com/science/journal/00109452

Pashler, H. (2000). Task switching and multitask performance. In: S. Monsell y J. Driver (Eds.). Control of Cognitive Processes: Attention and Performance XVIII. (pp. 277-307). Cambridge, MA: MIT Press.

Perry, R. J. y Hodges, J. R. (1999). Attention and executive deficits in Alzheimer's disease. Brain, 12, 383-404. doi:10.1093/brain/122.3.383

Posner, M. I. (1980). Orienting of attention. Q J Exp Psychol, 32(1), 3-25. doi:10.1080/00335558008248231

Posner, M. I. y Petersen, S. E. (1990). The attention system of the human brain. Anпu Rev Neurosci, 13, 25-42. doi:10.1146/annurev.neuro.13.1.25

Quintana, J. y Fuster, J. M. (1999). From perception to action: temporal integrative functions of prefrontal and parietal neurons. Cerebral Cortex, 9 , 213-221. doi:10.1093/cercor/9.3.213

Rapp, M., Krampe, R. y Baltes, P. (2006). Adaptive Task Prioritization in Aging: Selective Resource Allocation to Postural Control Is Preserved in Alzheimer Disease. Am J Geriatr Psychiatry, 14, 52-61. doi: 10.1097/01.JGP.0000192490.43179.e7

Reitan, R. M. (1958). Validity of the Trail Making Test as an indicator of organic brain damage. Percept Mot Skills, 8, 271-276. doi:10.2466/PMS.8.7.271-276

Rochon, E., Waters, G. S. y Caplan, D. (2000). The relationship between measures of working memory and sentence comprehension in patients with Alzheimer's disease. J Speech Lang Hear Res, 43(2), 395-413. Retrieved from http://islhr.asha.org/

Rogers, R. D. y Monsell, S. (1995). The cost of a predictable switch between simple cognitive tasks. J Exp Psychol Gen, 124, 207-231. doi:10.1037//0096-3445.124.2.207

Shimamura, A. P. (2002). Memory retrieval and executive control processes. In D. T. Stuss y R. T. Knight (Eds.). Principles of frontal lobe function (pp. 210-220). New York, NY, US: Oxford University Press. doi: 10.1093/acprof:oso/9780195134971.003.0013

Simon, J. R. (1969). Reactions towards the source of stimulation. I Exp Psychol, 81, 174-176. Retrieved from http://psycnet.apa.org/journals/xge/81/1/

Simone, P. M. y Baylis, G. C. (1997). Selective attention in a reaching task: effect of normal aging and Alzheimer's disease. J Exp Psychol Hum Percept Perform, 23, 595-608. doi:10.1037//0096-1523.23.3.595

Spieler, D. H., Balota, D. A. y Faust, M. E. (1996). Stroop performance in healthy younger and older adults and in individuals with dementia of the Alzheimer type. I Exp Psychol Hum Percept Perform, 22, 461-749. doi:10.1037//0096-1523.22.2.461

Stroop, J. R. (1935). Studies of interference in serial verbal reactions. $J$ Exp Psychol, 18, 643-62. doi:10.1037//0096-3445.121.1.15

Sullivan, M. P., Faust, M. E. y Balota, D. A. (1995). Identity negative priming in older adults and individuals with dementia of the Alzheimer type. Neuropsychology, 9, 537-555. doi:10.1037//0894-4105.9.4.537
Taylor, S. F., Kornblum, S., Lauber, E. J., Minoshima, S. y Koeppe, R. A. (1997). Isolation of specific interference processing in the Stroop task: PET activation studies. Neuroimage, 6, 81-92. doi:10.1006/nimg.1997.0285

Tipper, S. (1985). The negative priming effect: inhibitory priming by ignored objects. $Q J$ Exp Psychol, 37A, 571-590. doi: $10.1080 / 14640748508400920$

Tipper, S. P., Brehaut, J. C. y Driver, J. (1990). Selection of moving and static objects for the control of spatially directed action. J Exp Psychol Hum Percept Perform, 16, 492-504. doi:10.1037//0096-1523.16.3.492

Tirapu-Ustárroz, J., García-Molina, A., Luna-Lario, P., Roig-Rovira, T. y Pelegrín-Valero, C. (2008a). Modelos de funciones y control ejecutivo (I). Rev neurol, 46(11), 684-692. Retrieved from http://www.revneurol.com/

Tirapu-Ustárroz, J., García-Molina, A., Luna-Lario, P., Roig-Rovira, T. y Pelegrín-Valero, C. (2008b). Modelos de funciones y control ejecutivo (II) Rev neurol, 46(12), 742-750. Retrieved from http://www.revneurol.com/

Tirapu-Ustárroz, J., Muñoz-Céspedes, J. M. y Pelegrín-Valero, C. (2002). Funciones ejecutivas: necesidad de una integración conceptual. Rev neurol, 34(7), 673-685. Retrieved from http://www.revneurol.com/

Torralva, T., Roca, M., Gleichgerrcht, E., López, P. y Manes, F. (2009). INECO Frontal Screening (IFS). A brief, sensitive, and specific tool to assesss executive functions in dementia. J Int Neuropsychol Soc,1-10. doi: 10.1017/S1355617709990415

Traykov, L., Baudic, S., Thibaudet, M. C., Rigaud, A. S., Smagghe, A. y Boller, F. (2002). Neuropsychological deficit in early subcortical vascular dementia: comparison to Alzheimer's disease. Dement Geriatr Cogn Disord, 14(1), 26-32. doi:10.1159/000058330

Tse, C. S., Balota, D. A., Yap, M. J., Duchek, J. M., McCabe, D. P. (2010). Effects of Healthy Aging and Early Stage Dementia of the Alzheimer's Type on Components of Response Time Distributions in Three Attentional Tasks. Neuropsychology, 24, 300-315. doi: 10.1037/a0018274

Van der Linden, M., Collette, F., Salmon, E., Delfiore, G., Degueldre, C., Luxen, A. y Franck, G. (1999). The neural correlates of updating of information in verbal working memory. Memory, 7, 549-560. doi: 10.1080/096582199387742

Vaughan, F. L., Hughes, E. A., Jones, R. S., Woods, R. T. y Tipper S. P. (2006). Spatial negative priming in early Alzheimer's disease: evidence for reduced cognitive inhibition. J Int Neuropsychol Soc, 12(3), 416-423. doi: 10.1017/S1355617706060504

Verhaeghen, P. y Basak, C. (2005). Ageing and switching of the focus of attention in working memory: Results from a modified N-Back task. QJ Exp Psychol, 58A(1), 134-154. doi:10.1080/02724980443000241

Verhaeghen, P., Cerella, J., Bopp, K. L. y Basak, C. (2005). Aging and varieties of cognitive control: A review of meta-analyses on resistance to interference, coordination and task switching, and an experimental exploration of age-sensitivity in the newly identified process of focus switching. In R. W. Engle, G. Sedek, U. von Hecker y D. N. McIntosh (Eds.). Cognitive Limitations in Aging and Psychopathology: Attention, Working Memory, and Executive Functions. (pp. 160-189). Cambridge, MA: Cambridge University Press.

Verhaeghen, P. y De Meersman, L. (1998). Aging and the Stroop effect: metaanalysis. Psychol Aging, 13, 120-126. doi: 10.1037/08827974.13.1.120

Wang, K., Liang, M., Wang, L., Tian, L., Zhang, X., Li K. y Jiang, T. (2007). Altered functional connectivity in early Alzheimer's disease: A restingState fMRI study. Hum Brain Mapp, 28, 967-978. doi: $10.1002 / \mathrm{hbm} .20324$

Wilkinson, D. T., Halligan, P. W., Marshall, J. C., Büchel, C. y Dolan, R. J. (2001). Switching between the forest and the trees: brain systems in volved in local/global changed-level judgements. Neuroimage, 13, 56-67. doi:10.1006/nimg.2000.0678

Yeung, N., Cohen, J. D. y Botvinick, M. M. (2004). The neural basis of error detection: Conflict monitoring and the error-related negativity. Psychological Review, 111, 931-959. doi: 10.1037/0033-295X.111.4.931

(Artículo recibido: 29-03-2011, revisión: 01-09-2011, aceptado: 1-09-2012) 